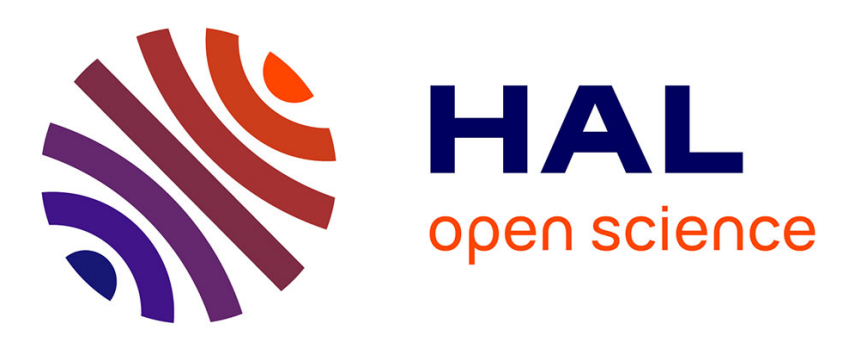

\title{
Ab Initio Batch Emulsion Thiol-Ene Photopolymerization
}

Cuong Minh Quoc Le, Marc Schmutz, Abraham Chemtob

\section{To cite this version:}

Cuong Minh Quoc Le, Marc Schmutz, Abraham Chemtob. Ab Initio Batch Emulsion Thiol-Ene Photopolymerization. Macromolecules, 2020, 10.1021/acs.macromol.0c00265 . hal-02538946

\section{HAL Id: hal-02538946 https://hal.science/hal-02538946}

Submitted on 9 Apr 2020

HAL is a multi-disciplinary open access archive for the deposit and dissemination of scientific research documents, whether they are published or not. The documents may come from teaching and research institutions in France or abroad, or from public or private research centers.
L'archive ouverte pluridisciplinaire HAL, est destinée au dépôt et à la diffusion de documents scientifiques de niveau recherche, publiés ou non, émanant des établissements d'enseignement et de recherche français ou étrangers, des laboratoires publics ou privés. 


\title{
$\mathrm{Ab}$ Initio Batch Emulsion Thiol-Ene
}

\section{Photopolymerization}

\author{
Cuong Minh Quoc Le, ${ }^{1,2}$ Marc Schmutz ${ }^{3}$ and Abraham Chemtob ${ }^{1,2^{*}}$ \\ ${ }^{1}$ Université de Haute-Alsace, CNRS, IS2M UMR7361, F-68100 Mulhouse, France \\ ${ }^{2}$ Université de Strasbourg, France \\ ${ }^{3}$ Institut Charles Sadron, CNRS, UPR 22, University of Strasbourg, 23 Rue du Loess, BP 84047, \\ 67034 Strasbourg, Cedex 2, France
}

* To whom correspondence should be addressed: abraham.chemtob@uha.fr, +33 389608834.

\begin{abstract}
Very few step polymer latexes have been produced by aqueous emulsion polymerization, and never with a clear picture of the operating mechanism. Therefore, finding new means to expand this widely process industrially beyond chain radical polymerization is highly desirable. Herein, we report the successful photoinitiated thiol-ene radical polyaddition of an emulsion based on two bifunctional monomers diallyl phthalate and 2,2-(ethylenedioxy)diethanedithiol. After 20 min irradiation $\left(\lambda_{\max }=385 \mathrm{~nm}, 3.7 \mathrm{~mW} \mathrm{~cm}^{-2}\right)$, polymerization has resulted in a conversion above $99 \%$, a coagulum-free poly(thioether) latex with a solids content of $10 \mathrm{wt} \%$, a number-average
\end{abstract}


molecular weight of $14 \mathrm{kDa}$, and an average particle diameter of $60 \mathrm{~nm}$. Reaction kinetics, particle size distribution, molecular weight progress have been studied in detail. To clarify the mechanism dictating particle formation, the number of particles per unit volume of water has been determined depending on reaction time, surfactant and initiator concentration. The nucleation mode is found to depart significantly from a conventional chain radical emulsion polymerization. Polymer particle formation mainly proceeds through the precipitation of oligoradicals (homogeneous nucleation) even when the concentration of surfactant exceeds its critical micellar concentration. The end of nucleation (Interval I) occurs until a monomer conversion of about $95 \%$. The dispersion's solids content can reach as high as $40 \%$ solids content, and the molecular weights remain reasonable $(>4 \mathrm{kDa})$ even if the functional groups are stoichiometrically imbalanced. In contrast to a conventional thermally induced polymerization, temporal control is demonstrated through sequential "on"-“off" cycles, and tuning of molecular weights is achieved by precise energetic dosage of radiation. This new step photopolymerization in emulsion thus opens an avenue for the synthesis of linear polysulfide latex without high energy homogenization and at low irradiance.

\section{For Table of Contents use only}

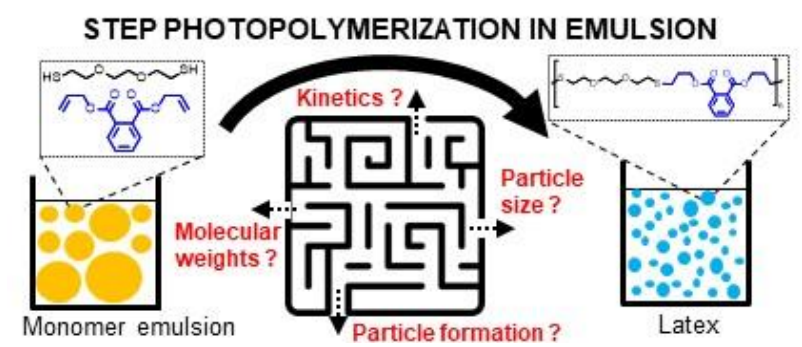




\section{INTRODUCTION}

Polymerization in dispersed systems is one of the most used techniques for the production of

polymers. ${ }^{1}$ A significant example is emulsion polymerization, a widely used process industrially that results in polymer dispersions with a typical average diameter in the $50-200 \mathrm{~nm}$ range. ${ }^{2-4}$ Compared to solution polymerization, emulsion polymerization involves much more mechanistic events that govern the rate of formation and growth of polymer particles. ${ }^{5,6}$ Over the last 70 years, understanding and predicting these events has been a major challenge for the academic community, with the goal of controlling the multiple characteristics of dispersed polymers: particle size and molecular weight distribution, polymer composition and architecture, etc. ${ }^{7,8}$ The complex nature of emulsion polymerization has not hindered the large scale use of "emulsion polymers" in many different industrial fields including coatings, adhesives, inks and non-woven textiles. ${ }^{9}$ Today, emulsion polymerization is still at the forefront in the field of eco-efficient production of polymers. Its sustained development is ensured by new environmental regulations and some key technical advantages of latex products including low viscosity, high molecular weights, heat removal capacity and water as possible continuous phase, etc. ${ }^{10}$ However, all current commercial emulsion polymers (styrene-butadiene, acrylic, vinyl acetate...) are exclusively prepared by chain radical polymerization carried out in tank reactors using thermal or redox radical initiator. ${ }^{11}$ Although further progress should be made in the future, all advances will necessarily be limited by the boundaries imposed by the mechanism of chain radical polymerization.

Very few step polymers - mostly polyesters - could be prepared by aqueous emulsion polymerization, ${ }^{12-16}$ and never with a clear picture of the operating mechanism. To achieve highmolecular-weight products by step polymerization, a number of experimental criteria must be 
fulfilled that are difficult to reconcile with an emulsion polymerization in water: very high monomer conversions (> 98\%), the absence of side reactions (to avoid stoichiometric imbalance of functional groups), and an efficient removal of the by-products (to shift the equilibrium in the direction of the polymer). ${ }^{17,18}$ In this context, miniemulsion polymerization has proven to be better suited than emulsion polymerization for the synthesis of step polymers in water because mass transfer of monomer through aqueous phase is avoided, thus decreasing the risk of side reactions. ${ }^{19}$ Using this technique, step polymerization in miniemulsion gave rise to a number of latexes based on epoxy,${ }^{20}$ polyurethane,${ }^{21}$ polyester ${ }^{22}$ poly(dimethylsiloxane),${ }^{23}$ polysulfide ${ }^{24,25}$ and a broad range of waterborne "step polymer-acrylics" hybrids. ${ }^{26}$ However, by comparison with a (macro)emulsion, the preparation of a monomer miniemulsion adds complexity and cost in the form higher energy consumption and equipment investment. ${ }^{27}$ This explains why this technique has not yet undergone a large-scale industrial implementation. ${ }^{28}$ Currently, the preferred method towards waterborne step polymer dispersions still involves a solution polymerization in non-aqueous media, followed by redispersion in water through ionic groups attached to the polymer backbone or external surfactants. ${ }^{29}$

Emulsion polymerization being the workhorse process for the production of dispersed polymers, further research is therefore required to overcome the technical challenges of emulsion step polymerization. ${ }^{30}$ In a seminal work, Shipp et al. ${ }^{31}$ reported in 2017 a promising example of radical-mediated thiol-ene step polymerization in emulsion. Starting from polyfunctional thiol and alkene monomer emulsions, submicron-sized poly(thioether) particles with linear or crosslinked structure were achieved using a thermal radical initiator. Compared to other step polymerizations, thiol-ene polyaddition features indeed several characteristics able to push up molecular weights such as high conversions, absence of by-product and no side-reaction with 
water. $^{32,33}$ Although a significant amount of experimental data was reported in this aforementioned study, issues related to polymerization kinetics, particle size distribution and molecular weight progress are still poorly understood, particularly with regard to differences with the well-studied chain radical polymerization in emulsion. Additionally, the fundamental mechanisms that dictate particle formation in a step radical polymerization in emulsion need to be further clarified.

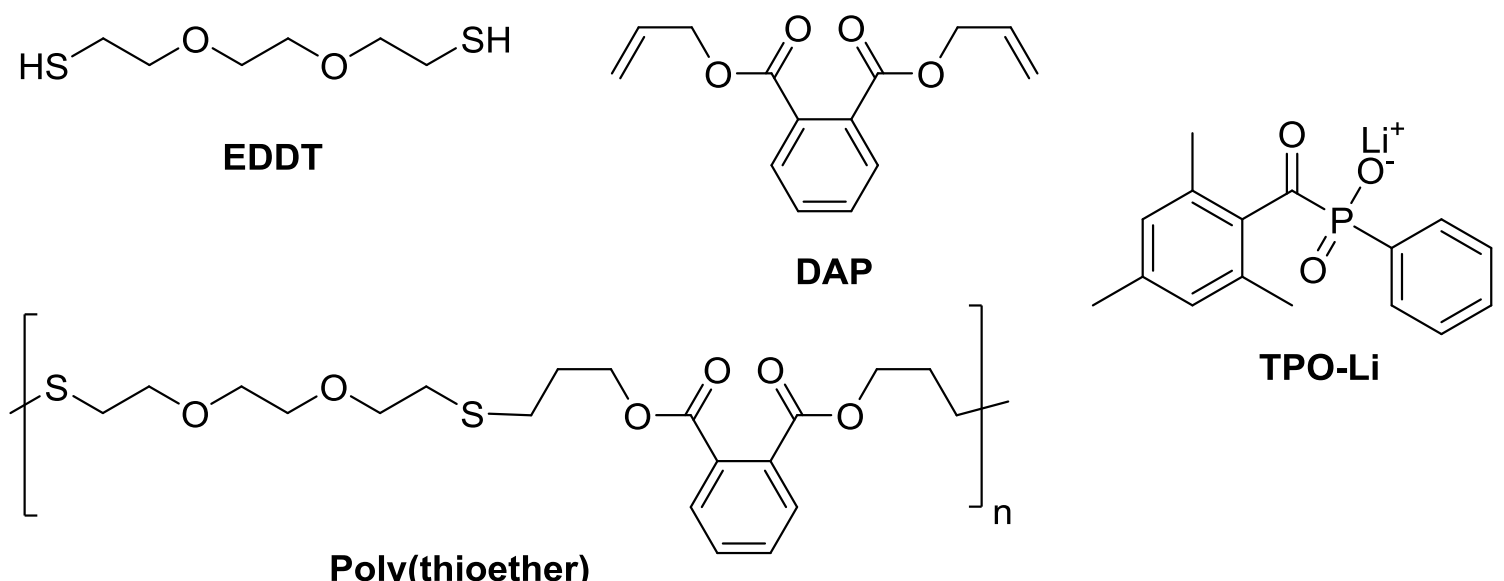

Scheme 1. Chemical structure of diene and dithiol monomers DAP and EDDT respectively, watersoluble radical photoinitiator (TPO-Li), and poly(thioether) derived from EDDT-DAP thiol-ene polyaddition. Photolysis mechanism and absorption spectrum of TPO-Li are shown in Fig. S1 of SI.

To address these questions, we have studied the batch photoinduced thiol-ene polyaddition of an aqueous surfactant-stabilized emulsion prepared with the bifunctional monomers diallyl phthalate (DAP) and 2,2-(ethylenedioxy)diethanedithiol (EDDT) $\left(1: 1\right.$ equiv.) at $25^{\circ} \mathrm{C}$ (Scheme 1). To be very close to the operational conditions of a conventional emulsion polymerization, we have used sodium dodecyl sulfate (SDS) as the surfactant and a water-soluble photoinitiator lithium phenyl(2,4,6-trimethylbenzoyl)phosphinate (TPO-Li). Photopolymerization $\left(\lambda_{\max }=385\right.$ $\mathrm{nm}, 3.7 \mathrm{~mW} \mathrm{~cm}^{-2}$ ) has resulted after $20 \mathrm{~min}$ in a conversion of monomers higher than $99 \%$, a coagulum-free poly(thioether) latex with a solids content of $10 \mathrm{wt} \%$, and stable particles having 
an average diameter of $60 \mathrm{~nm}$. Despite significant radiation attenuation due to the multiple scattering of micron-sized monomer droplets, faster polymerization rates and higher molecular weights were achieved compared to a control experiment involving a water-soluble redox initiator. To clarify the nucleation mechanism, we have determined the number of particles per unit volume of water $\left(N_{p}\right)$ as function of conversion, surfactant concentration and initiator concentration. The fact that our emulsion step polymerization is photochemically initiated opens up new possibilities for improving temporal control of reaction and tuning molecular weight tunable through irradiance. To prove the versatility of our process, the effect of latex solids content $(5-40 \mathrm{wt} \%)$ and non-stoichiometric thiol-ene ratio have been also studied.

\section{EXPERIMENTAL SECTION}

\section{Materials}

Diallyl phthalate (DAP, > 98\%), 2,2-(ethylenedioxy)diethanedithiol (EDDT, > 97\%), DLdithiothreitol (DTT, > 98\%), 2,5-di-tert-butylhydroquinone (DBHQ, > 98\%), sodium dodecyl sulfate (SDS, 98\%), lithium phenyl(2,4,6-trimethylbenzoyl)phosphinate (TPO-Li, > 99\%) and diphenyl(2,4,6-trimethylbenzoyl)phosphine oxide (TPO, > 98\%) were purchased from TCI Chemicals. Water-insoluble dye Orasol Blue 855 also referred to as Solvent Blue 70 was obtained from Kremer Pigmente GmbH. Other chemicals include L-ascorbic acid (Sigma Aldrich, 99\%), hydrogen peroxide (Carlo Erba, 30\%) and iron (II) sulfate heptahydrate (Merck, > $80 \%$ ). Dimethyl sulfoxide- $d_{6}\left(\mathrm{DMSO}-d_{6}, 99.5 \% \mathrm{D}\right)$ was bought from Eurisotop. HPLC grade tetrahydrofuran (THF) was received from VWR. All emulsion polymerizations used deionized water. All chemicals were used without further purification. 


\section{Synthesis}

Photoinitiated thiol-ene emulsion polymerization. A photolatent organic phase composed of a

1:1 stoichiometric mixture of thiol and ene functional groups was prepared by adding EDDT (1.70 g, 9.2 mmol, 1 equiv.), DAP (2.30 g, 9.2 mmol, 1 equiv.) and DBHQ (40.5 mg, 0.178 mmol, 0.02 equiv.) to a $10 \mathrm{~mL}$ vial. $1 \mathrm{~g}$ of the resultant organic solution was then transferred to a $20 \mathrm{~mL}$ soda-lime glass vial (external diameter: $25 \mathrm{~mm}$, height: $70 \mathrm{~mm}$ ). To this latter was added an aqueous phase composed of TPO-Li (20 mg, $0.067 \mathrm{mmol}, 0.03$ equiv.), SDS (35 mg, $3.5 \mathrm{wt} \%$ with respect to monomers, $13.5 \mathrm{mM}$ in water) and $9 \mathrm{~mL}$ of deionized water. A coarse emulsion with an organic phase content of $10 \mathrm{wt} \%$ was prepared using Ultra-Turrax ${ }^{\circledR}$ T25 homogenizer (IKA-Werke) at $20000 \mathrm{rpm}$ for $5 \mathrm{~min}$. Alternatively, a magnetic stirrer (RCT-basic from IKAWerke) can be used to form the monomer emulsion upon operating at $1100 \mathrm{rpm}$ for $20 \mathrm{~min}$. In both cases, the monomer droplets were relatively unstable and coalesced if agitation was stopped causing a phase separation. Thus, photopolymerization was performed immediately after emulsion preparation at room temperature inside a LED circular photochemical reactor. The photoreactor was constructed by winding a $385 \mathrm{~nm}$ LED strip (SMD3528, 60 LED/Meter, LightingWill, length: $1000 \mathrm{~mm}$ ) around a quartz cylinder (internal diameter: $80 \mathrm{~mm}$, length: 100 $\mathrm{mm}$ ). Irradiation was maintained for $20 \mathrm{~min}$, while the vial was kept under continuous magnetic stirring $(1100 \mathrm{rpm})$. The vial was placed centrally in the photoreactor where it received at its surface an irradiance $(I)$ of $3.7 \mathrm{~mW} \cdot \mathrm{cm}^{-2} . I$ value was measured by a calibrated silicon photodiode radiometer (S120VC, Thorlabs). At given irradiation times, $50 \mu \mathrm{L}$ aliquots were withdrawn of the vial for analysis of monomer conversion, particle size and molecular weight.

Redox initiated thiol-ene emulsion polymerization of EDDT-DAP. A similar DAP-EDDT monomer emulsion was produced upon replacing the water-soluble photoinitiator in the aqueous 
continuous phase by a mixture of ascorbic acid (16.5 mg, $0.094 \mathrm{mmol}, 0.04$ equiv.) and $\mathrm{FeSO}_{4}$ (0.49 mg, 0.003 mmol, 0.0014 equiv.) acting as reductant and catalyst respectively. ${ }^{34}$ The polymerization was initiated by introducing $9.7 \mu \mathrm{L}$ of a $30 \%$ wt $\mathrm{H}_{2} \mathrm{O}_{2}$ solution $(0.094$ mmol, 0.04 equiv.) into the $20-\mathrm{mL}$ vial containing the coarse emulsion. The reaction was stirred by magnetic stirring at $1100 \mathrm{rpm}$ for $20 \mathrm{~min}$.

Emulsion photopolymerization in presence of a water-insoluble dye to determine particle nucleation mode. This experiment was adapted from a publication of Chern et al. ${ }^{35,36}$ In a variant procedure of the emulsion photopolymerization described above, Orasol Blue 855 (20 mg) was initially dissolved into the organic phase. Given the potential role of hydrophobic agent played by the extremely hydrophobic dye, magnetic stirring was used to prepare the thiol-ene monomer emulsion, and avoid the formation of a miniemulsion. Control experiments demonstrated that transport of dye through the aqueous was not possible, as also proved separately by Chern et al. ${ }^{35}$ Consequently, the dye was initially distributed among the monomer droplets and the micelles when present. After polymerization, its location depends on the nucleation mode. With homogeneous nucleation as the only operating mechanism, the dye cannot be encapsulated inside the polymer particle and a dye precipitate suspended throughout the liquid is formed. Conversely, occurrence of micellar nucleation is manifested by a fraction of dye incorporated inside the particles. The weight percentage of dye incorporated into the particles $\left(P_{d y e}\right)$ was determined by UV-Vis spectroscopy at $672 \mathrm{~nm}$. This wavelength corresponds to a maximum absorption peak of the dye, where no other component of the latex absorbs. It was verified that the dye had a minimal impact on the course of the polymerization and the latex characteristics. After irradiation, the latex was allowed to stand at room temperature for 3 days to favor the sedimentation of the insoluble dye fraction at the bottom of the dispersion. The rest of the 
dispersion was filtered through a $0.45 \mu \mathrm{m}$ PVDF membrane to remove the rest of the precipitated dye. Subsequently, an aliquot $(100 \mu \mathrm{L})$ of the filtered latex was then dissolved in $2.9 \mathrm{~mL}$ of DMSO to form a homogeneous solution that was analyzed subsequently by UV-Vis spectroscopy. $P_{d y e}$ was determined using a calibration curve established through a set of standard dye samples $\left(0-10 \mu \mathrm{g} \mathrm{mL}^{-1}\right)$ of known concentration in DMSO. A theoretical $P_{d y e}^{\text {th }}$ was calculated assuming that all micelles are nucleated. ${ }^{35}$

$$
P_{d y e}^{t h}(\%)=100 \times S_{\text {monomer }}^{\text {micelle }} \times([S D S]-C M C) \times V_{\text {water }} \times\left(\frac{m_{E D D T}}{M_{E D D T}}+\frac{m_{D A P}}{M_{D A P}}\right)
$$

Where:

- $S_{\text {monomer }}^{\text {micelle }}$ is the molar solubility of monomer in SDS micelles (mol per mol of SDS). A value of $1.7 \mathrm{~mol}$ of monomer dissolved in micelles per mol of SDS was used for calculation. This value corresponds to the tricomponent system benzene/SDS/ $\mathrm{H}_{2} \mathrm{O} .^{37}$

- $[S D S]$ and $C M C$ are respectively the SDS molar concentration (M). A CMC value of $8.2 \mathrm{mM}$ at $25^{\circ} \mathrm{C}$ was used. ${ }^{37}$

- $V_{\text {water }}$ is the volume of water (L).

- $M_{E D D T}$ and $M_{D A P}$ are the molar masses of EDDT and DAP mixture $\left(\mathrm{g} \mathrm{mol}^{-1}\right)$.

- $m_{E D D T}$ and $m_{D A P}$ are the monomer weights $(\mathrm{g})$.

\section{Characterization}

Dynamic light scattering (DLS). Particle sizes were measured by DLS using a VASCO particle size analyzer (Cordouan technologies) operating at $658 \mathrm{~nm}$ laser. The intensity of the scattered light was detected at an angle of $135^{\circ}$. Latex sample was diluted 100 times in SDS solution (13.5 $\mathrm{mM}$ ) to obtain a final concentration of $0.1 \mathrm{wt} \%$. NanoQ software version 2.6 was employed for processing DLS data. Dilution avoids multiple scattering and allows the residual monomer to diffuse out the polymer particles. (Water solubility of EDDT at $25^{\circ} \mathrm{C}$ is around $15 \mathrm{~g} \mathrm{~L}^{-1}$ and DAP 
at $20{ }^{\circ} \mathrm{C}$ is $\left.0.18 \mathrm{~g} \mathrm{~L}^{-1}\right)$. Consequently, a dilution factor of 100 times is sufficient to drain almost all of the monomer out of the particle. Therefore, the measured particle size corresponds to that of the non-swollen polymer particle and not the remaining monomer droplets for aliquots taken at partial conversions (swelling by oligomers cannot be estimated). Each sample was analyzed six times. Only samples containing baseline error below $1 \%$ were considered. The cumulants analysis method was used to extract intensity-average diameter (z-average) and polydispersity index $\mathrm{PdI}^{\mathrm{DLS}}$. The z-average value values were used to calculate the number of latex particles.

$$
N_{p}=\frac{6 \times m_{\text {monomer }} \times x}{\pi \times D_{Z}^{3} \times V \times \rho_{p}}
$$

Where $x$ is the ene monomer conversion, $\mathrm{D}_{z}$ the z-average diameter, $V$ the volume of the continuous phase (water) and $\rho_{\mathrm{p}}$ the density of polymer (1.13).

Using the particles refractive index, the inverted Mie algorithm was applied to determine a number-average diameter $D_{n}$ and a volume-average diameter $D_{v}$. The goal was to put DLS-based and TEM-based measurements on a common basis to make a comparison possible. ${ }^{38}$

Nuclear magnetic resonance (NMR). Evolution of ene and thiol conversions during the reaction was monitored by ${ }^{1} \mathrm{H}-\mathrm{NMR}$ and ${ }^{13} \mathrm{C}-\mathrm{NMR}$ spectroscopy. All NMR spectra were recorded in DMSO- $d^{6}$ on a Varian $300-$ MR. All chemical shifts were reported in parts per million (ppm) relative to the residual DMSO- $d^{6}(\delta 2.50 \mathrm{ppm})$. At least 64 scans were recorded for each spectrum. Typically, a $30 \mu \mathrm{L}$ aliquot was charged into an NMR tube containing $570 \mu \mathrm{L}$ of DMSO- $d^{6}$ and the spectrum recorded. For each trial, ene conversion was determined upon comparing the integration area of resonances of methylene protons attached to a vinyl group $\left(-\mathrm{CH}=\mathrm{CH}_{2}\right)$ in DAP at $4.75 \mathrm{ppm}$ and that of the same methylene protons in the polymer at 4.25 ppm. Thiol conversion was calculated based on the change in the integral value for the sulfhydryl proton at $1.58 \mathrm{ppm}$ at the beginning and after a selected time (see Fig. S2 in SI for details). For 
the analysis of the poly(thioether), water was evaporated from the latex, and the solid was then cleaned with methanol and dried under vacuum.

Size exclusion chromatography (SEC). Number-average molecular weights $\bar{M}_{n}$ and weightaverage molecular weights $\bar{M}_{w}$ were measured by SEC (Agilent 1260 Infinity) containing a G1314B variable wavelength detector operating at $254 \mathrm{~nm}$, a G7800A multidetector suite consisting of a refractive index detector and a viscosimeter detector, and using THF as eluent. The solvent was pumped through a set of columns (Polymer Laboratories ResiPore, nominal particle size: $3 \mu \mathrm{m}$; porosity: $2 \mu \mathrm{m})$ composed of a guard column $(50 \times 7.5 \mathrm{~mm})$ and two columns $(300 \times 7.5 \mathrm{~mm})$. The flowrate was set at $1 \mathrm{~mL} \mathrm{~min}^{-1}$ and the column temperature was controlled at $35{ }^{\circ} \mathrm{C}$. The system was then calibrated using calibration standards (EasiVial polystyrene standards from Agilent). A portion of $50 \mu \mathrm{L}$ latex sample was placed into a vial and water was removed by a nitrogen flow. The residue was rinsed with $0.5 \mathrm{~mL}$ of methanol and subsequently with $0.5 \mathrm{~mL}$ of water. The purified latex was dried under vacuum at room temperature for 2 hours. The mass of polymer in vial was recorded before dissolution in THF. The volume of THF was adjusted to give a final concentration $5 \mathrm{mg} \mathrm{mL}^{-1}$ and the polymer solution in THF was filtered through a $0.2 \mu \mathrm{m}$ PTFE membrane before injection. Agilent GPC/SEC software and multi-detector were used to obtain the molecular weight data.

UV-Vis absorption. Absorption spectra were recorded on a V730 UV/vis spectrometer from Jasco with a bandwidth of $1 \mathrm{~nm}$ and a scan speed of $1000 \mathrm{~nm} \min ^{-1}$. All the solutions were analyzed in a quartz cuvette of $1 \mathrm{~cm}$ length.

Cryo-TEM analysis. The vitrification of the samples was carried out in a homemade vitrification system. The chamber was held at $22{ }^{\circ} \mathrm{C}$ and the relative humidity at $80 \%$. A $5 \mu \mathrm{L}$ 
drop of the sample (1-2 $\left.\mathrm{mg} \cdot \mathrm{mL}^{-1}\right)$ was deposited onto a lacey carbon film covered grid ((Ted Pella, Redding, CA, USA) rendered hydrophilic using an ELMO glow discharge unit (Cordouan Technologies, Bordeaux, France). The grid was automatically blotted to form a thin film which was plunged in liquid ethane held at $-190{ }^{\circ} \mathrm{C}$ by liquid nitrogen. That way, a vitrified film was obtained, in which the native structure of the particles was preserved. The grid was mounted onto a cryo holder (Gatan 626, Pleasanton, CA, USA) and observed under low dose conditions in a Tecnai G2 microscope (FEI, Eindhoven, Netherland) at $200 \mathrm{kV}$. Images were acquired using an Eagle slow scan CCD camera (FEI). The number-average diameters $\left(D_{n}\right)$ and volume-average diameters $\left(D_{v}\right)$ were calculated using the following equations:

$$
\begin{aligned}
& D_{n}=\frac{\sum n_{i} D_{i}}{\sum n_{i}} \\
& D_{v}=\frac{\sum n_{i} D_{i}^{4}}{\sum n_{i} D_{i}^{3}}
\end{aligned}
$$

where $n_{i}$ designates the number of particles of diameter $D_{i}$. A minimum of 700 particles were taken into for the calculations. Polydispersity index $\operatorname{PdI}^{\mathrm{TEM}}=\left(\sigma / D_{n}\right)^{2}$ is defined as the squared ratio of the standard deviation $(\sigma)$ of the particle diameter distribution divided by the number-average diameters. PdI ${ }^{\mathrm{TEM}}$ was used to estimate the width of size distribution.

\section{RESULTS AND DISCUSSION}

\section{Reaction Kinetics, Particle Size and Molecular Weight Progress}

The thiol-ene photopolymerization of a conventional oil-in-water emulsion requires specific conditions of implementation: bicomponent and micro-sized EDDT/DAP monomer droplets $(1: 1$ 
equiv.), a water-soluble photoinitiator (TPO-Li), and an aqueous continuous phase containing a surfactant (SDS) at a concentration above the CMC. For ensuring effective temporal control, the starting emulsion must also be chemically stable before exposure to irradiation, which is generally a challenge with thiol-ene formulations. ${ }^{39-41}$ Di(tert-butyl)-1,4-hydroquinone (DBHQ), a typical radical inhibitor, was thus added to the DAP-EDDT organic phase (0.02 equiv.). This was found to be an efficient means to prevent premature thiol-ene emulsion polymerization, and maintain a minimum chemical stability of $2 \mathrm{~h}$. Without stabilizer, ene conversion was nearly 60 $\%$ after emulsification, whether the initiator was present or not. This result lends confidence that for this thiol-ene couple, the instability is mainly assigned to a radical mediated thiol-ene reaction. The precise mechanism accounting for the spontaneous generation of radicals in this complex biphasic system shall be discussed in a separate publication. To compensate for the inhibitor presence, an appropriate excess of photoinitiator (0.03 equiv.) was used.

Starting with a DBHQ-stabilized DAP-EDDT emulsion, the ene conversion-time curves in Figure 1 support the photolatency of monomer droplets not exposed to UV radiation (plot a) and the role of light ( $385 \mathrm{~nm}$, monochromatic LED) as the main trigger of polymerization (plot $\mathbf{b}$ ). Even at low irradiance $\left(3.7 \mathrm{~mW} \mathrm{~cm} \mathrm{~cm}^{-2}\right)$, a conversion of approx. $99 \%$ was achieved in less than 5 min. However, continuous irradiation was maintained for 20 min because it is known that step polymerization shows the presence of high-molecular-weight polymers only near the very end of the reaction. Despite the inhibitor, no induction period was visible because its duration may be too short to be detected by an offline technique such as ${ }^{1} \mathrm{H}$ NMR spectroscopy (used here to monitor the concentration of monomer in the reaction vessel). Remarkably, the very high backscattering signal caused by the emulsified micron-sized droplets of monomer did not prevent high polymerization rates (see backscattering values before and after irradiation in Fig. S3 of SI). 
In addition, precise measurement of reflectance revealed a significant decrease after polymerization. The fact the reaction medium becomes less scattering is consistent with the disappearance of large monomer droplets by diffusion through the aqueous phase into much smaller polymer particles of lower scattering efficiency. ${ }^{42}$ The thiol conversion-time curve was difficult to obtain because the sulfhydryl protons of EDDT could not be quantified without ambiguity by ${ }^{1} \mathrm{H}$ NMR when conversions become large. However, a mass polymerization involving only the thiol-ene reactants, clearly established the synchronicity of thiol and ene functional groups' conversion (Fig. S4 in SI). The similarity of thiol and ene kinetic data support that allyl ether cannot undergo homopolymerization, and that the predominant reaction is thiolene radical addition. In agreement with this, ${ }^{1} \mathrm{H}$ and ${ }^{13} \mathrm{C}$ NMR analysis of the polymer latex confirmed that the polymer backbone exhibits a high density of thioether units (Fig. S5, SI).

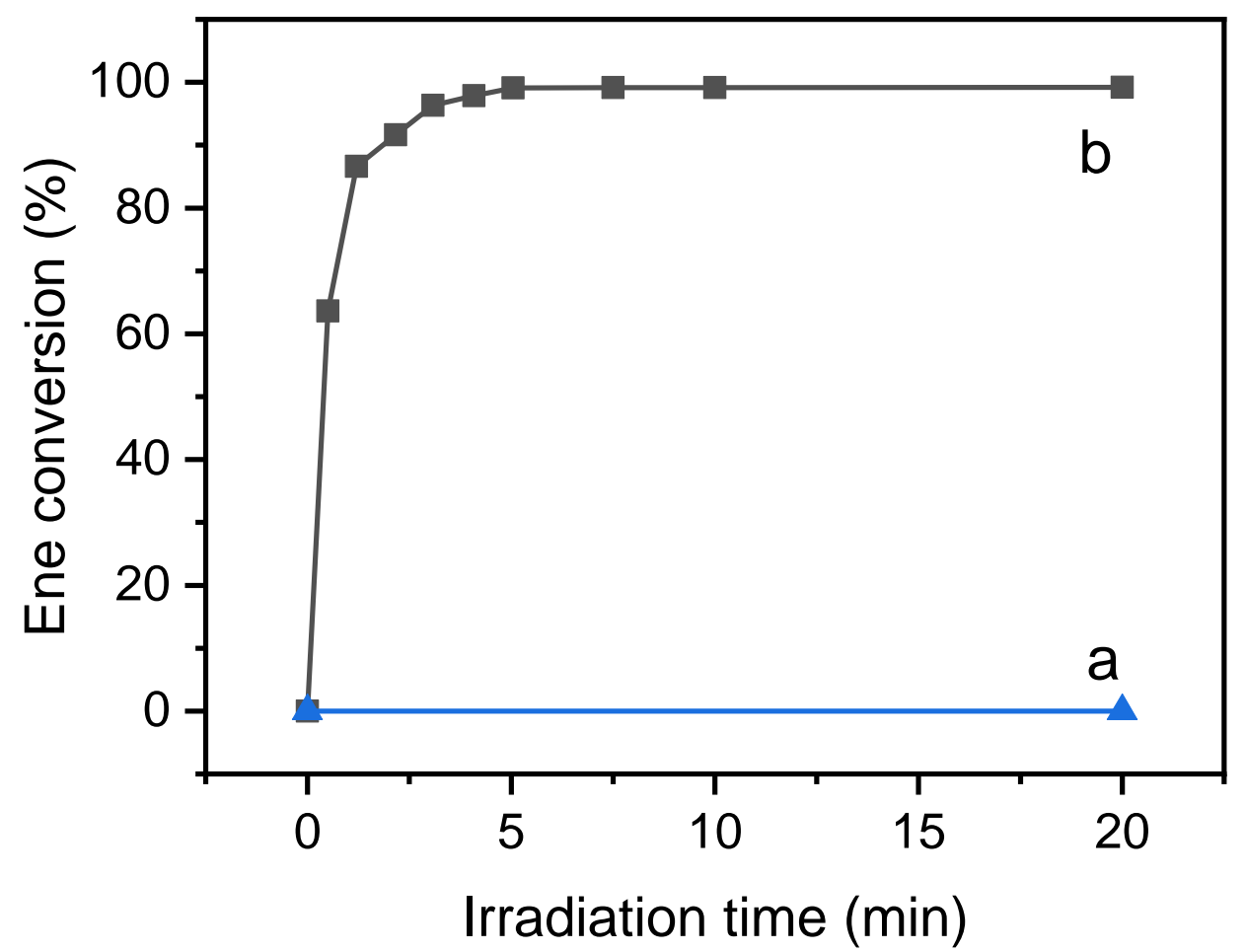

Figure 1. Conversion-time curves for EDTT-DAP emulsion photopolymerization. Plot a: without irradiation; plot b: monochromatic irradiation at $385 \mathrm{~nm}, I=3.7 \mathrm{~mW} \mathrm{~cm}^{-2}$. Irradiation was performed in a LED circular photochemical reactor (see experimental section for details). 
Direct imaging of the polymer particles using microscopy required the use of cryo-TEM. A first attempt with conventional TEM showed that the soft polysulfide particles had coalesced upon drying due to their sub-ambient glass transition temperature $\left(-40{ }^{\circ} \mathrm{C}\right.$, see DSC trace in Fig S6). ${ }^{43}$ As shown in Figure 2A, the latex is almost monomodal and near-spherical in shape. The particles display a number-average diameter of $62 \mathrm{~nm}$, that is customary in emulsion polymerization, and a relatively broad size distribution $\left(\mathrm{PdI}^{\mathrm{TEM}}=0.14\right)$. The intensity-average diameter (z-average) obtained from DLS analysis was significantly higher $(98 \mathrm{~nm})$, while the size dispersity remained in the same order of magnitude $\left(\mathrm{PdI}^{\mathrm{DLS}}=0.15\right)$. DLS measures typically show larger sizes than found in microscopic studies. ${ }^{44}$ The fact that the sample is not monodisperse accounts for this discrepancy. With a size range spanning $25-125 \mathrm{~nm}$, the largest particles have a scattering cross-section 4 orders of magnitude greater than the smallest ones. Therefore, intensity-average value is further weighted towards the minor population of larger particles due to their higher scattering efficiency. Using Mie theory (see experimental section for details), it was possible to calculate number-average and volume-average distributions, making possible the comparison between DLS- and TEM-based data. Figure 2B shows on the same plot the excellent agreement between the two methods as regards with volume and number distribution analysis. Process replicability was also verified as shown by a standard deviation for intensity-average diameters lower than $5 \mathrm{~nm}$ (see Table $\mathbf{S 1}$ in SI). The process is also robust since the water-soluble photoinitiator can be added either before or after the preparation of the monomer emulsion with no impact on reaction rates and particle size. 

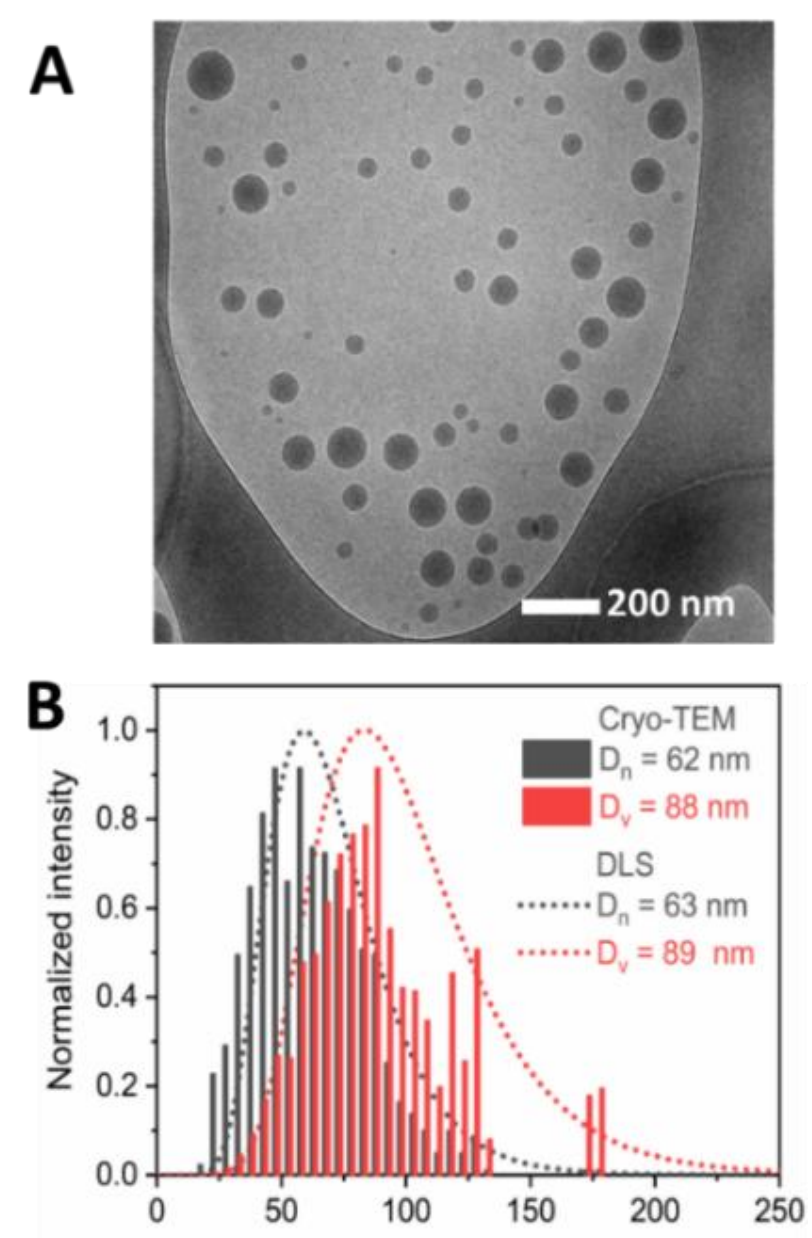

Figure 2. A: Cryo-TEM image of a latex obtained by EDDT-DAP emulsion photopolymerization. B: Volume-average and number-average distributions obtained from cryo-TEM-based measures (bars) and determined from DLS data (dashed line) after conversion of intensity distribution through Mie theory. $D_{n}$ and $D_{v}$ are respectively number-average and volume-average diameters.

Figure 3A shows the evolution of number-average molecular weight $\left(\bar{M}_{n}\right)$ and molecular weight dispersity $\left(\mathrm{Ð}=\bar{M}_{w} / \bar{M}_{n}\right)$ as function of ene-conversion. At the end of irradiation (20 min), the molecular-weight distribution was relatively broad $(\bigoplus=2.8)$, and a maximum $\bar{M}_{n}$ value of $14,000 \mathrm{~g} \mathrm{~mol}^{-1}$ was achieved. This latter value is reasonable from the practical viewpoint and given the difficulty of obtaining high-molecular-weight polymers in a step polymerization. Significant increase in molecular weights was reported near the very end of the reaction $(>97 \%$ conversion), consistently with a step polymerization. However, the polymerization slightly 
departs from the normal step pathway as revealed a comparison with Carothers equation (Figure 3B). In our case, a higher number-average degree of polymerization was obtained at moderate percent of conversion $(60-95 \%)$. The underlying reason is unclear at this time although such evolution was observed in certain step polymerizations, which involves fast reactions rates and separation of monomers in two phases. ${ }^{45,46}$ Under these conditions, monomers can react preferentially at the interface with the growing polymer chain ends instead of creating new chains, thus driving higher molecular weights. It is hypothesized that such interfacial polymerization may occur in our system, primarily dictated by the high rate constants of thiol-ene reaction and distinct partitioning of EDDT and DAP in aqueous phase and polymer particles based on their different solubility in water (EDDT has a water solubility about 80 times higher than DAP). The fact that almost complete conversion was achieved with a $1: 1$ stoichiometric ratio suggests that partitioning effects are not overly significant. However, further study is necessary to understand these results. 

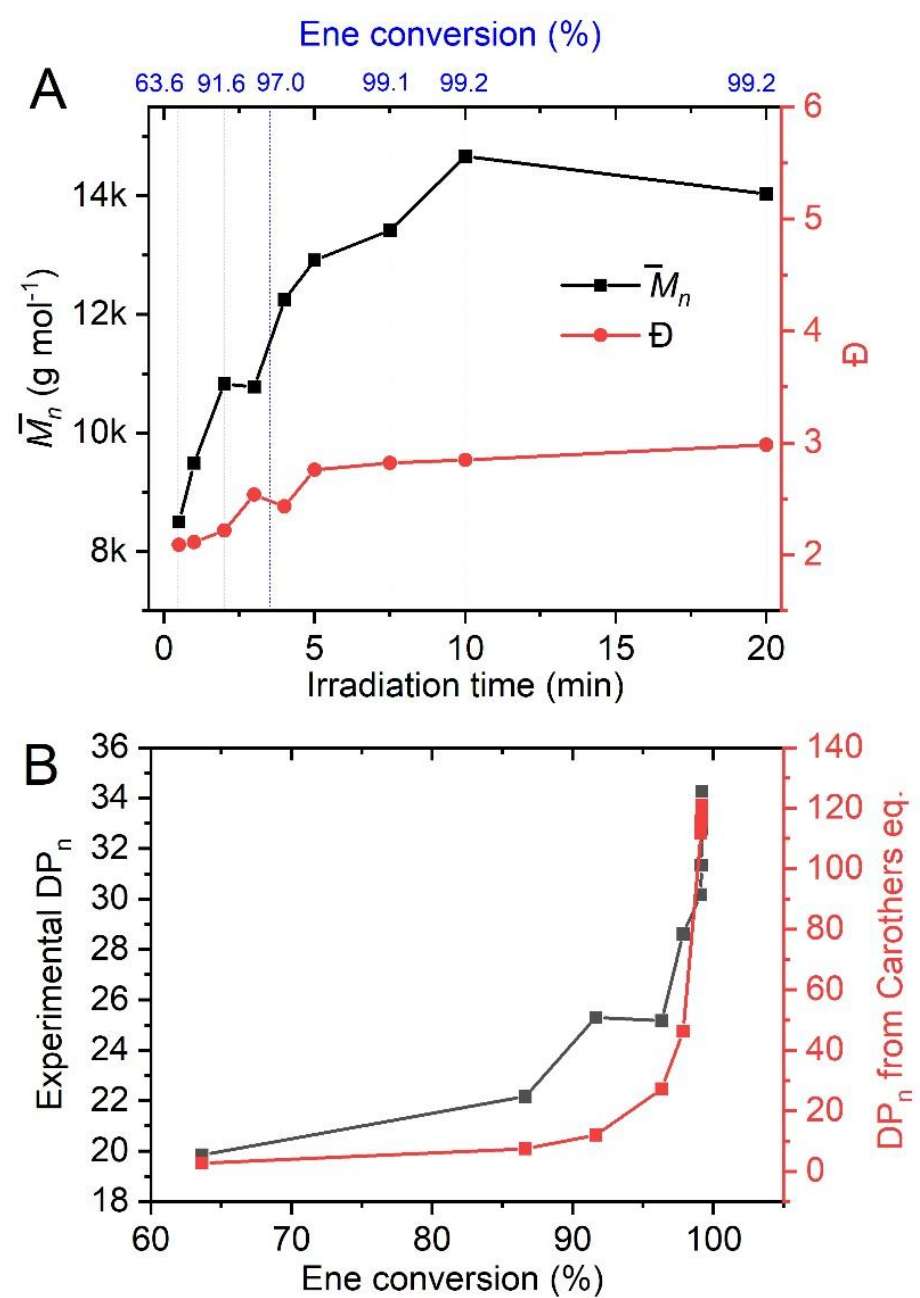

Figure 3. A: Dependence of number-average molecular weight $\left(\bar{M}_{n}\right)$ and dispersity (Đ) on monomer conversion and irradiation time for emulsion photopolymerization of EDDT-DAP at $25^{\circ} \mathrm{C}$. The vertical blue lines show the corresponding ene conversion at a specific irradiation time. B: Comparison between experimental number-average degree of polymerization $\left(\mathrm{DP}_{\mathrm{n}}\right)$ and theoretical values derived from Carothers equation $\left(\mathrm{DP}_{\mathrm{n}}=1 /(1-\mathrm{Conv}\right.$.) for two monomers in equimolar quantities) as a function of ene conversion.

A salient feature of thiol-ene polymerization compared to chain-growth polymerization is its insensitivity to oxygen inhibition. ${ }^{47}$ This was firstly confirmed by unchanged polymerization kinetics and particle size when purging the reactor with nitrogen to flush out oxygen (Table S2, SI). On the other hand, lower $\bar{M}_{n}$ values levelling off at approx. $10300 \mathrm{~g} \mathrm{~mol}^{-1}$ were systematically obtained in photopolymerization under nitrogen atmosphere. We postulated that atmospheric oxygen might cause a loss of thiol groups by thiol air oxidation. Although the net result of a stoichiometric imbalance between the functional groups is normally a decrease the 
degree of polymerization, the opposite trend was found in our case. This is probably due to the formation of thiyl radical-terminated chain ends by air oxidation and their subsequent bimolecular coupling reaction to yield disulfide bonds. The validity of this assumption was assessed by exposing the two latexes produced under air and nitrogen to a reducing solution containing DL-dithiothreitol (DTT). As shown in Figure 4A, only the latex produced under air showed a reduction of molecular weight from 13500 to $9300 \mathrm{~g} \mathrm{~mol}^{-1}$ and a narrowed molecular weight distribution $(Đ=2.2)$. Moreover, a close inspection of the ${ }^{1} \mathrm{H}-\mathrm{NMR}$ spectra in DMSO- $d^{6}$ (Figure 4B) revealed that only this latex displayed disulfide-adjacent methylene protons (-S-S$\mathrm{CH}_{2}-\mathrm{CH}_{2}$ ) appearing as a distinctive triplet at 2.83 ppm. After treatment with DDT, this resonance almost vanished, thereby providing evidence of S-S bonds in the original polymer backbone. Conversely, the resonance assigned to monosulfide-adjacent methylene protons (-S$\mathrm{CH}_{2}-\mathrm{CH}_{2}$ ) - resulting from thiol-ene addition and arising at $2.68 \mathrm{ppm}$ - was not affected by DTT in both cases. In all polymers, the triplet peak from the terminal thiol protons (1.58 ppm) was also not detected, consistently with the high ene conversion ( $>99 \%)$ determined by NMR. Consequently, it is not possible to assume for a reaction performed in ambient conditions that the initial thiol-allyl stoichiometric ratio is the effective stoichiometric ratio throughout the polymerization from start to finish. However, a quantitative analysis of the polymer ${ }^{1} \mathrm{H}$ and ${ }^{13} \mathrm{C}$ NMR spectra reveals that an average polymer chain contains $99 \%$ thioether monomer and only $1 \%$ of dilsulfide linkages. Except for the occasional presence of SS bonds, thiol-ene polymerization with no possibility of chain transfer yields only linear poly(thioether) chains without branching, resulting in the formation of crystalline domains (see DSC trace in Fig. S6). Therefore, the final polymer composition and structure are well-defined because only dictated by the alternating sequence of DAP and EDDT units. 

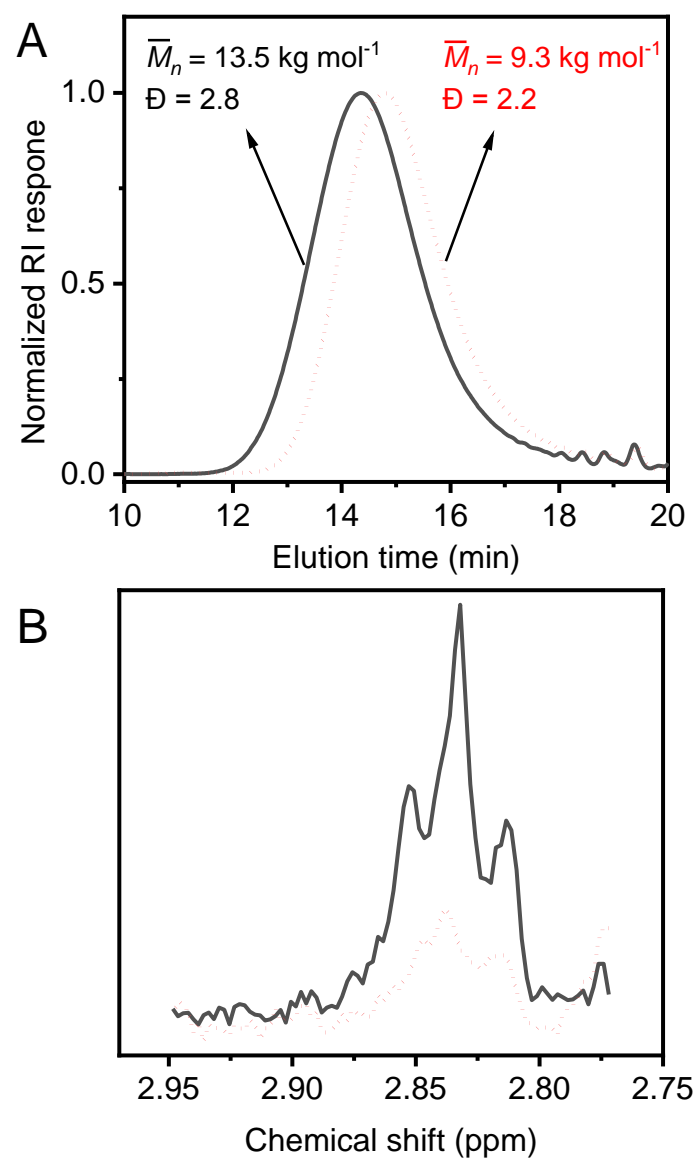

Figure 4. Effect of reducing agent (DTT) on the SEC profile (A) and ${ }^{1} \mathrm{H}$ NMR spectrum in DMSO- $d^{6}$ (B) of a poly(thioether) latex produced under air by EDDT-DAP emulsion photopolymerization. Solid line: as-synthesized latex. Dotted line: the same latex after treatment with DDT (excess amount of DTT in DMSO- $d_{6}, 12 \mathrm{~h}$ at room temperature). The NMR spectrum shows the $2.75-2.95 \mathrm{ppm}$ region with the triplet resonance of the disulfide-adjacent methylene protons $\left(-\mathrm{S}-\mathrm{S}-\mathrm{CH}_{2}-\mathrm{CH}_{2}\right)$ centered at $2.83 \mathrm{ppm}$.

\section{Fundamentals of Particle Formation}

A detailed understanding of the mechanism that governs particle formation in emulsion thiolene photopolymerization is crucial. Particle formation has been a long-studied research area but only for emulsion chain polymerization. ${ }^{1,2,5}$ However, step and chain polymerizations differ significantly, in particular in the dependence of polymer molecular weight and monomer concentration on conversion. Hence, a significant departure from the well-known emulsion polymerization mechanism is expected for a thiol-ene step polymerization. Regardless of the 
polymerization mechanism, for an ab initio system, there is an initial period in which the particle number is changing due to particle formation, which is referred to as interval I. Two principle nucleation processes account for the formation of particles: micellar nucleation in which the entry of an oligo-radical in a micelle produces a new polymer particle; and homogeneous nucleation in which radicals react in the aqueous phase with solubilized monomer to form growing oligomeric species. These species will form particles when the critical water solubility length is reached. Micellar nucleation often prevails above the CMC, while homogeneous nucleation is the single operative mechanism possible when micelles are absent. Though the subject of nucleation is very complex, a significant amount of mechanistic information can be obtained in our system by examining the behavior of $N_{p}$ (obtained from intensity-average diameters) as a function of monomer conversion, surfactant concentration and initiator concentration.

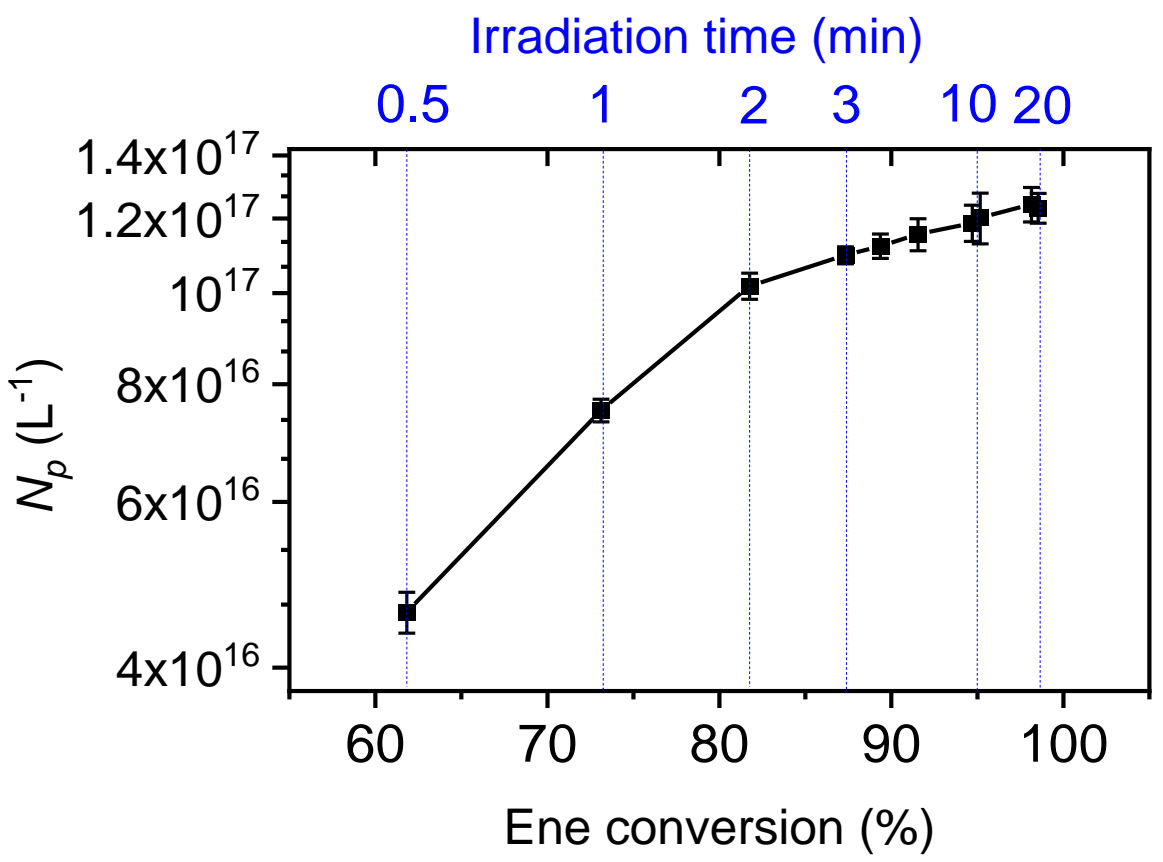

Figure 5. Evolution of $N_{p}$ versus monomer conversion (or reaction time) during EDDT-DAP emulsion photopolymerization. Irradiation at $385 \mathrm{~nm}, I=0.9 \mathrm{~mW} \mathrm{~cm}{ }^{-2}, 20 \mathrm{~min}$. The vertical blue lines show the corresponding irradiation time for each ene conversion point. 
Figure 5 shows the evolution of the number of polymer particles produced in the course of the reaction as a function of DAP conversion. A 4-fold decrease of irradiance was chosen for this experiment $\left.(I=0.9 \mathrm{~mW} \mathrm{~cm})^{-2}\right)$ to slowdown polymerization rates and obtain more experimental points at the early stage of reaction. It can be seen that $N_{p}$ shows a rapid increase until $80 \%$ conversion, then the rate formation of new particles decreases very quickly until $97-98 \%$ conversion to reach almost steady-state value during the last percent of conversion (see data in Table S3, SI). When particles concentration is high, the probability of radical capture by a formed particle becomes much greater than the new particle formation, which accounts for the less efficient particle formation at longer times. While this observation has been well reported, a more puzzling feature is the long nucleation period that extends up to $97-98 \%$ conversion. By contrast, interval I is generally the shortest interval for a chain polymerization in emulsion, its duration varying in the typical range $2-15 \%$ conversion. The long nucleation time can be reconciled with the specific molecular weight evolution in a step polymerization: a slow molecular weight rise and high-molecular-weight polymers achieved only near the very end of the reaction. Whichever the mode, nucleation requires nucleation requires either surface-active oligoradicals (z-mer) or insoluble oligomers (j-crit), both achieved through addition of a sufficient number of monomer units (z-mer). Because chain size and the amount of polymer are dependent on conversion in step polymerization, obtaining an entire population of insoluble or surface active radicals is predicted to occur more slowly and progressively than in a chain polymerization. This causes a longer nucleation time, which also accounts for the relatively broad size distribution of the latex. In addition, it is also possible that a minimum time (induction time) exists to obtain polymer particles. Indeed, no particles can be formed before the oligomer radicals 
have reached a critical chain length, and this may take longer time for a step polymerization. Due to a fast polymerization, our first experimental aliquot at $30 \mathrm{~s}$ corresponds already to more than $60 \%$ conversion, and it was not possible to verify this hypothesis.

Figure 6A and 6B show respectively the effect of surfactant and initiator concentration on the final number of particles. Upon varying [SDS] from 2 to $100 \mathrm{mM}$, a first regime at low SDS concentration ( $2-7 \mathrm{mM}$, below $\mathrm{CMC})$ is observed where the number of particles that can stabilized is dependent on the total surface area of surfactant available in the system (itself proportional to the surfactant concentration). The order of dependence of $N_{p}$ on [SDS] is 2.58 , and particles are formed by homogeneous nucleation because no micelles can be formed. Above $7 \mathrm{mM}$, which is close to the CMC value in water $(8.2 \mathrm{mM})$, the system has reached steady state with regard to $N_{p}$, and the number of particles remain relatively insensitive to the concentration of added surfactant. When micellar nucleation plays a significant role, $\mathrm{N}_{\mathrm{p}}$ depends on surfactant concentration above CMC and tends to increase with [S]. In our case, no increase in $N_{p}$ or slope change was observed as the CMC was exceeded, which is generally the case when micellar nucleation adds or replaces homogeneous nucleation. This supports the minor role played by micellar nucleation and that a similar nucleation mode may occur below and above CMC. Therefore, homogeneous nucleation may be thus regarded as the prevailing nucleation mode in this system regardless of the surfactant concentration. A broad correlation exists between homogeneous nucleation and the likelihood of solution polymerization in the aqueous phase. In our system, solution polymerization should be favored relative to polymerization in the particles due to slow molecular weight evolution and the high water-solubility of EDDT (increasing also the probability for radicals to be desorbed from the particles). To emphasize the importance of solution polymerization, TPO-Li was changed by TPO, a water-insoluble analogue. A latex with 
a much greater diameter in the micrometer size range was created (see data in Table S2), indicating the central role played by initiation in aqueous phase and a very different particle formation mechanism.

Figure 6B shows the dependence of $N_{p}$ as a function of initiator concentration, keeping SDS concentration unchanged at $13.5 \mathrm{mM}$. $N_{p}$ was found to be directly dependent on the rate of radical generation. This trend is expected whatever the mechanism of particle formation and polymerization. In the DAP-EDDT system, $N_{p}$ varies with the 0.21 power of photoinitiator concentration, which is relatively small. When extensive radical desorption occurs due to more water-soluble oligo-radicals, a large fraction of nucleation is initiated by desorbed radicals with the results that $N_{p}$ is generally little affected by initiator concentration.
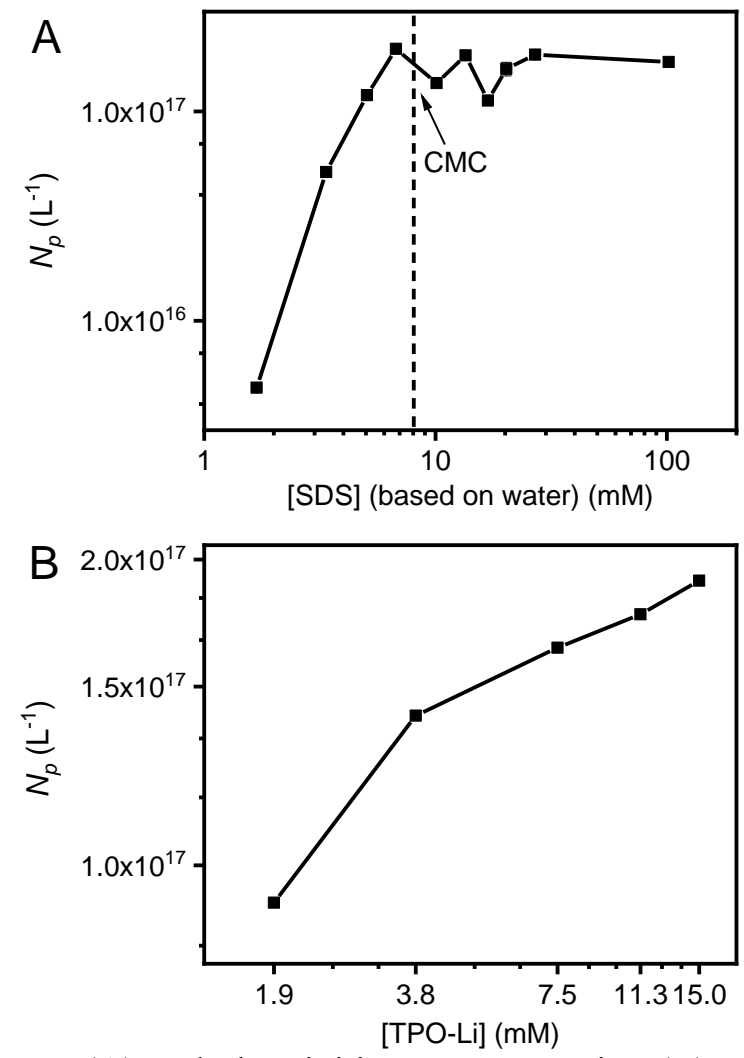

Figure 6. Effect of surfactant (A) and photoinitiator concentration $(\mathbf{B})$ on number of particles per unit volume during EDDT-DAP emulsion photopolymerization. Irradiation at $385 \mathrm{~nm}, I=0.9 \mathrm{~mW} \mathrm{~cm}{ }^{-2}, 20$ $\min$. 
In order to confirm the dominant role played by homogeneous nucleation, a polymerization was carried out with a thiol-ene monomer phase containing a water-insoluble dye (Orasol Blue 855, see structure in Fig. S7). Below CMC or when homogeneous nucleation is the only mechanism accounting for particle formation, the percent of dye incorporated in the final latex $\left(P_{d y e}\right)$ is predicted to be very small and independent of surfactant concentration because the extremely hydrophobic dye is unable to diffuse from monomer droplets to particles. Figure 7 is the variation of $P_{d y e}$ (monitored by UV spectroscopy) as a function of emulsifier concentration. Very significantly, it should be noted that there is no discontinuity of $P_{d y e}$ when surfactant concentration exceeds CMC. In addition, the values of $P_{d y e}$ are small $(0.6 \%)$ for the whole range of surfactant concentrations studied, and consistent with literature values when a single homogeneous nucleation operates. ${ }^{35,36}$ A small fraction of droplet nucleation or occasional droplet-particle coalescence events may account for the small deviation from zero. For comparison, the theoretical $P_{d y e}^{t h}$ in the case of a complete micellar nucleation was plotted on the same graphic (see calculation details in the experimental section). In this case, a linear increase above $\mathrm{CMC}$ is expected due to a higher concentration of monomer-swollen micelles. By contrast, the steady state with regard to $P_{d y e}$ supports homogeneous nucleation as the predominant mechanism of particle formation. 


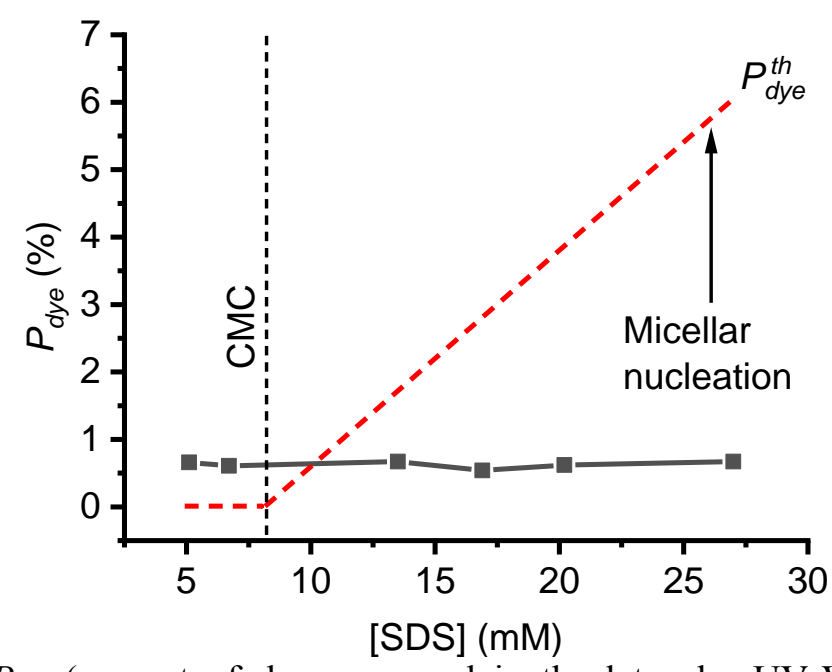

Figure 7. Variation of $P_{d y e}$ (percent of dye measured in the latex by UV-Vis spectroscopy) as a function of SDS concentration for the EDDT-DAP emulsion photopolymerization performed in presence of Orasol blue dye. $P_{d y e}^{t h}$ is an estimation of the percent of dye in particles considering that all micelles are converted into particle.

\section{Key Features of Emulsion Thiol-ene Photopolymerization}

Photochemically initiated thiol-ene emulsion polymerization has several advantageous features, that are successively highlighted in the following section: (i) temporal control of reaction, (ii) molecular weight tunable by irradiance, (iii) latex functionalization, (iv) high solids content latex, and (v) lower particle size and higher molecular weights compared with conventional redox initiators.

i. Temporal control of EDTT-DAP emulsion polymerization was investigated by switching on/off the irradiation source at different times over the course of the reaction. The instant on/off capacity of LED is indeed a distinctive advantage compared to conventional UV arc radiation sources. As can be seen in Figure 8, monomer conversion occurred only when the reactor was placed under UV exposure, while an almost complete and instantaneous interruption was observed when the irradiation was stopped. This distinctive feature is of interest to limit the risk of runaway reaction whereas the continuous generation of radicals cannot be stopped in a thermal 
polymerization due to thermal inertia. Keeping the same light dose as for continuous irradiation, similar monomer conversion (>99\%) and intensity-average diameter $(95 \mathrm{~nm})$ were achieved.

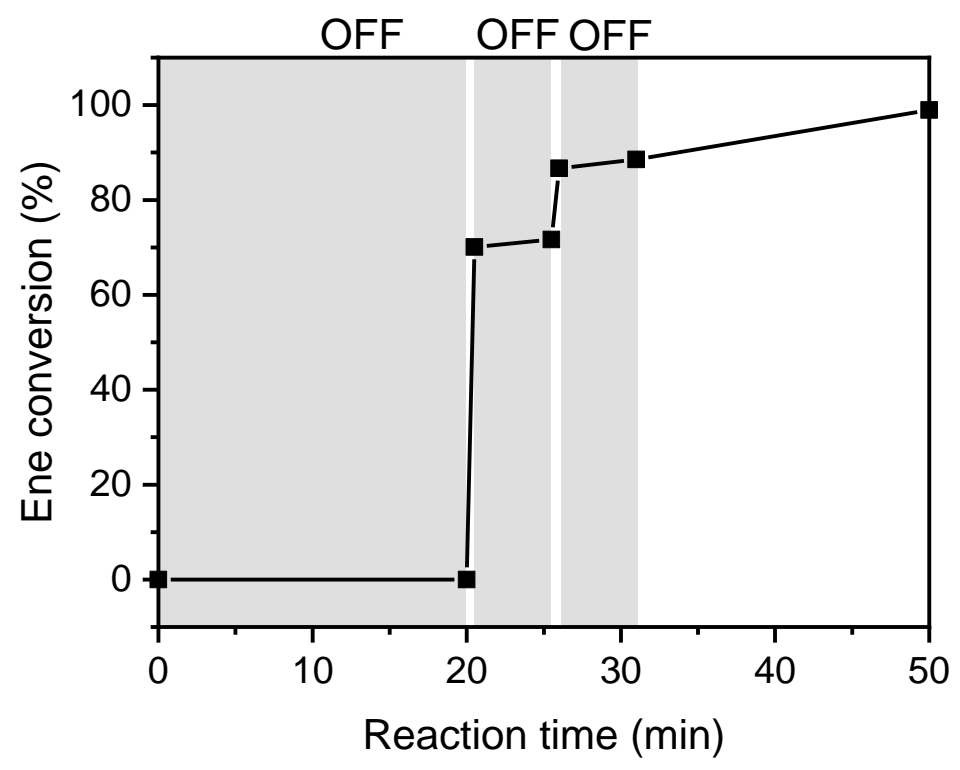

Figure 8. Effect of discontinuous irradiation on ene conversion-time for DAP-EDDT emulsion photopolymerization. Grey areas correspond to periods of non UV exposure while the white areas the system was exposed to radiation for $30 \mathrm{~s}$. Irradiation was performed in a LED circular photochemical reactor: $\lambda_{\max }=385 \mathrm{~nm}, I=3.7 \mathrm{~mW} \cdot \mathrm{cm}^{-2}$.

ii. Figure 9A is the evolution of molecular weight as a function of irradiance. At very low irradiance $\left(0.4 \mathrm{~mW} \mathrm{~cm}^{-2}\right)$, only moderate molecular weights were found $\left(3,800 \mathrm{~g} \mathrm{~mol}^{-1}\right)$. The main reason is probably that irradiation time of $20 \mathrm{~min}$ is not sufficient, leading to low monomer conversion (34\%) and large particle sizes $(>600 \mathrm{~nm})$. In the $3.7-9 \mathrm{~mW} \mathrm{~cm}^{-2}$ irradiance range, an almost full conversion ( $>99 \%$ ) could be reached after $20 \mathrm{~min}$. While particle sizes were slightly changed (90 - $120 \mathrm{~nm}$, see Table S1), number-average molecular weights progressively increased from 7,000 to $13,000 \mathrm{~g} \mathrm{~mol}^{-1}$. This trend illustrates the dependence of molecular weight on the concentration of initiating radicals, and demonstrates the ability to control the molecular weight by tuning irradiance. It is possible that slightly greater conversions achieved at higher irradiance (not easily detectable by ${ }^{1} \mathrm{H}$ NMR) account for the higher $\bar{M}_{n}$ values found experimentally. 
iii. Another critical feature of thiol-ene step growth polymerization is its potential to control functional groups on the surface of the particles by using off-stoichiometric monomer feed ratios. Figure 9B shows the variation of molecular weight depending on thiol:ene stoichiometric ratio. As expected, a stoichiometric imbalance in ene or thiol caused a drastic decrease of $\bar{M}_{n}$ from 13,500 to $6,400 \mathrm{~g} \mathrm{~mol}^{-1}$ with a narrowing of dispersity from 2.6 to 1.9 . Functionalization is therefore coupled to molecular weight, which is less desirable than if functionalization could be achieved independently. Nevertheless, polymers could be formed despite a significant difference in stoichiometry of functional groups. This result provides further support for an interfacial polymerization mechanism that is much less dependent on stoichiometry than a solution or mass polymerization.
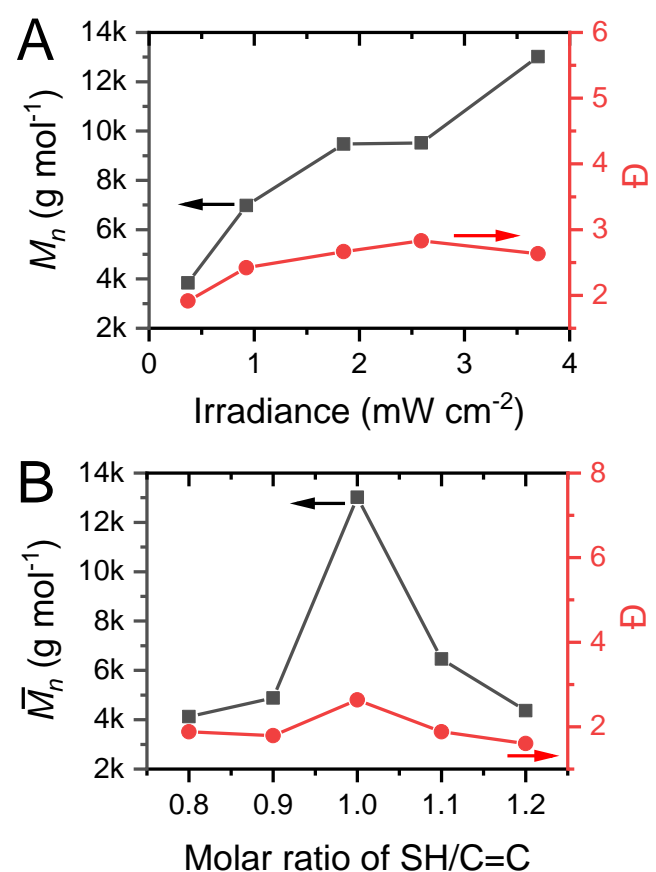

Figure 9. The influence of UV irradiance (A) and thiol:ene stoichiometric ratio (B) on polymer molecular weight. In A, a 1:1 thiol-ene ratio was used; in B, an irradiance of $3.7 \mathrm{~mW} \cdot \mathrm{cm}^{-2}$ was chosen, in both cases the irradiation time at $365 \mathrm{~nm}$ was $20 \mathrm{~min}$. 
iv. All previous photopolymerizations targeted latexes with a solids content of 10 wt $\%$ (weight of solid material/weight of latex). However, much higher solids contents are generally desired for latex based on chain polymerization to maximize the reactor production and minimize transport costs. ${ }^{48}$ To evaluate the flexibility of our process, the organic phase content was varied from 5 to $40 \mathrm{wt} \%$ while keeping surfactant concentration at $3.5 \mathrm{wt} \%$ (with respect to monomers) and the irradiation conditions unchanged. The effect on particle size and molecular weights are shown in Figure 10. Particle diameter gradually increased from 80 to $160 \mathrm{~nm}$ at higher solids content, but without noticeable variation in particle size distribution $\left(\mathrm{PdI}^{\mathrm{DLS}}=0.18\right)$ and conversion (>98-99\%). Conversely, molecular weight was lowered to $8,500 \mathrm{~g} \mathrm{~mol}^{-1}$. It is likely that at higher solids content, slightly lower conversions were obtained, thus requiring probably more irradiation time to achieve higher molecular weights. However, one must emphasize the possibility to produce a latex with a high solids content (40 $\mathrm{wt} \%)$, reasonable molecular weights, without residual monomer (intrinsic property of a step polymerization) and no coagulum.

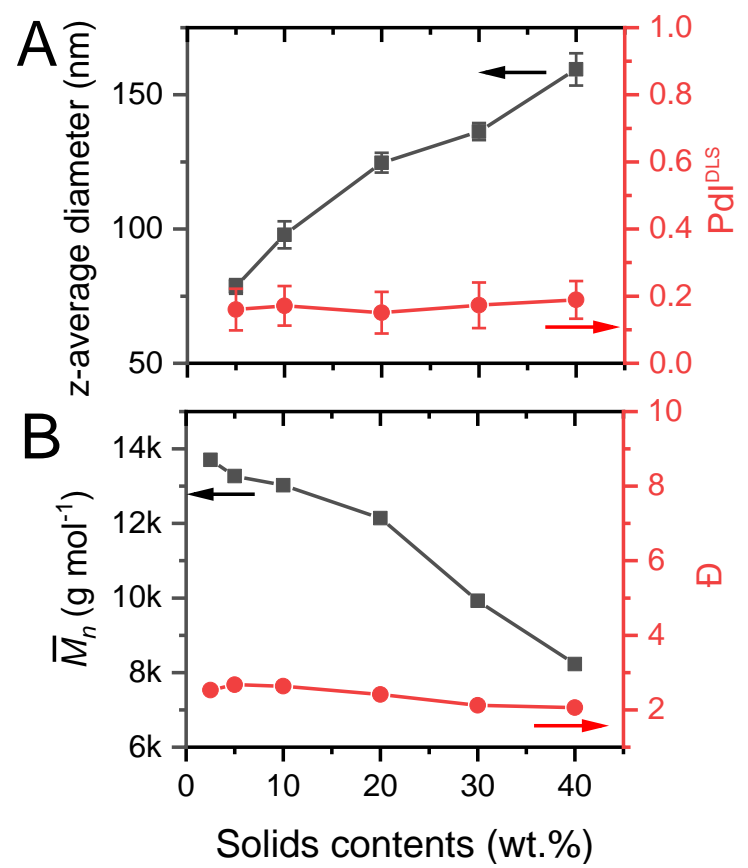

Figure 10. A: Effect of solids content on particle average diameter (z-average values) and size distribution $\left(\mathrm{PdI}^{\mathrm{DLS}}\right)(\mathrm{A})$; B: Effect of solids content on molecular weight $\left(\bar{M}_{n}\right)$ and polydispersity (Đ). 
v. Conventional emulsion polymerization generally relies on thermal initiators operating at a minimum temperature of $75{ }^{\circ} \mathrm{C}$ for their decomposition. Conversely, water-soluble redox initiators can be exploited at room temperature, and make possible a comparison with our photoinitiated system since the polymerization can be carried out at $25^{\circ} \mathrm{C}$ in both cases. We used a tricomponent system based on hydrogen peroxide (oxidant) together with ascorbic acid (reductant) and ferrous ion (catalyst), that is a common water-soluble redox initiator. ${ }^{34}$ As can be seen in Table 1, redox initiation yielded broader particle size distribution $\left(\mathrm{PdI}^{\mathrm{DLS}}=0.22\right)$, much larger average-diameter $(422 \mathrm{~nm})$ and was marked by a 2-fold decrease in molecular weight $\left(6,800 \mathrm{~g} \mathrm{~mol}^{-1}\right)$ compared to TPO-Li photoinitiator. The difference of results can be interpreted on the basis of distinct rates of radical generation. Despite non-homogeneous and limited radiation distribution, it is expected that a photochemical initiation produces a higher rate of initiation, thus increasing the number of polymer particles formed. We should note that high particles numbers are associated with small particle size. Higher polymerizations rates can be driven by a higher number of particles in a photochemically induced polymerization. Conversely, radical generation by redox initiator may be less efficient at $25^{\circ} \mathrm{C}$, leading to smaller number of particles, and lower molecular weights.

Table 1. Effect of initiator on latex properties derived from EDDT-DAP (thiol:ene $=1: 1$ equiv.) emulsion photopolymerization performed at $25^{\circ} \mathrm{C}$. Irradiation at $385 \mathrm{~nm}, I=3.7 \mathrm{~mW} \mathrm{~cm}{ }^{-2}, 20 \mathrm{~min}$.

\begin{tabular}{|l|l|l|l|l|c|}
\hline \multicolumn{1}{|c|}{ Initiator } & \multicolumn{2}{|c|}{$\begin{array}{c}\mathrm{PdI}^{\mathrm{DLS}} \\
\mathrm{nm}\end{array}$} & $\begin{array}{c}\text { ene Conv. } \\
\%\end{array}$ & $\begin{array}{c}\bar{M}_{n} \\
\mathrm{~kg} \mathrm{~mol}^{-1}\end{array}$ & Đ \\
\hline TPO-Li & & & & \\
\hline Ascorbic acid $/ \mathrm{H}_{2} \mathrm{O}_{2} / \mathrm{Fe}^{2+\mathrm{b}}$ & $422 \pm 30$ & 0.17 & $>99$ & 13.5 & 2.8 \\
\hline
\end{tabular}

a 0.03 equiv. of the water-soluble radical photoinitiator TPO-Li was used, [TPO-Li] $=7 \mathrm{mM} .{ }^{b}$ The mixture of ascorbic acid $/ \mathrm{H}_{2} \mathrm{O}_{2} / \mathrm{Fe}^{2+}$ (0.04:0.04:0.001 equiv.) acts as a redox initiator. 


\section{CONCLUSION}

A systematic investigation on thiol-ene emulsion step photopolymerization in water at $385 \mathrm{~nm}$ was performed. Linear polymer nanoparticles with size lower than $100 \mathrm{~nm}$ could be synthesized from two bifunctional monomers EDDT and DAP using experimental conditions very close to a conventional chain emulsion polymerization. Compared to other step polymerizations carried out in aqueous emulsion, thiol-ene polymerization has the advantage of being able to simultaneously attain both high reaction rates and reasonable molecular weights $\left(\bar{M}_{n}>13,000 \mathrm{~g} \mathrm{~mol}^{-1}\right)$. In addition, this process is unique in that it affords the means of understanding how a stepwise polymerization mechanism changes the typical particle formation process. $N_{\mathrm{p}}$ calculations and a separate experiment using an extremely water-insoluble dye initially dissolved in the monomer phase showed independently that polymer particle formation mainly proceeds through the precipitation of oligo-radicals (homogeneous nucleation) even when the concentration of surfactant exceeds its critical micellar concentration (CMC). Additionally, interval I (nucleation) occurs until a monomer conversion of about $97-98 \%$, which is significantly longer than a typical emulsion polymerization mechanism. Like in a conventional chain mechanism, polymerization of the monomers in solution takes place but does contribute more significantly, since the molecular weights increase more slowly and propagating radicals precipitate out of aqueous solution for much higher conversions. Thus, the larger part played by solution polymerization may lead homogeneous nucleation to extend and become the predominant nucleation mode. This new emulsion thiol-ene photopolymerization process also exhibits a number of advantages: stable polysulfide latex can be produced without high energy homogenization, at low irradiance and up to $40 \mathrm{wt} \%$ solids content. The ability to harness the inherent advantages of light over heat for 
creating the initiating radicals has been exploited since temporal control and tuning reaction of molecular weight by irradiation intensity were demonstrated.

\section{ACKNOWLEDGEMENT}

This project has received funding from the European Union's Horizon 2020 research and innovation programme under the Marie Skłodowska-Curie grant agreement $\mathrm{N}^{\mathrm{o}} 765341$ (Project PHOTO-EMULSION, MSCA-ITN-2017).

\section{SUPPORTING INFORMATION}

The Supporting Information is available free of charge at https://pubs.acs.org/doi/10.1021/acs.macromolecules.9b02994. Additional characterization results including mechanism and absorption spectrum of the water-soluble photoinitiator TPO-Li (Fig S1), the backscattering signals of DAP-EDDT macroemulsion (Fig S2), an example of calculation of thiol-ene monomer conversion (Fig S3), conversion-time curves for the bulk polymerization of EDDT-DAP (Fig S4), ${ }^{1} \mathrm{H}-\mathrm{NMR}$ and ${ }^{13} \mathrm{C}-\mathrm{NMR}$ spectra of polysulfide latex (Fig S5), DSC thermogram of the poly(thioether) derived from emulsion polymerization (Fig S6), chemical structure of the dye (Fig S7), replicability experiments (Table S1), effect of photoinitiation addition mode and nitrogen purging on latex physico-chemical properties (Table S2) and evolution of $N_{p}$ versus monomer conversion (Table S3). 


\section{REFERENCES}

(1) Chemistry and Technology of Emulsion Polymerisation; van Herk, A. M., Ed.; John Wiley \& Sons Ltd: Oxford, UK, 2013.

(2) Chern, C. S. Emulsion Polymerization Mechanisms and Kinetics. Prog. Polym. Sci. 2006, 31 (5), 443-486.

(3) Gilbert, R. G. Emulsion Polymerization: A Mechanistic Approach; Academic Press, 1995.

(4) Antonietti, M.; Tauer, K. 90 Years of Polymer Latexes and Heterophase Polymerization: More Vital than Ever. Macromol. Chem. Phys. 2003, 204 (2), 207-219.

(5) Thickett, S. C.; Gilbert, R. G. Emulsion Polymerization: State of the Art in Kinetics and Mechanisms. Polymer 2007, 48 (24), 6965-6991.

(6) Sajjadi, S.; Brooks, B. W. Butyl Acrylate Batch Emulsion Polymerization in the Presence of Sodium Lauryl Sulphate and Potassium Persulphate. J. Polym. Sci. Part Polym. Chem. 1999, 37 (21), 3957-3972.

(7) Harkins, W. D. A General Theory of the Mechanism of Emulsion Polymerization1. J. Am. Chem. Soc. 1947, 69 (6), 1428-1444.

(8) Smith, W. V.; Ewart, R. H. Kinetics of Emulsion Polymerization. J. Chem. Phys. 1948, 16 (6), 592-599.

(9) Chern, C.-S. Principles and Applications of Emulsion Polymerization; John Wiley \& Sons, 2008.

(10) Asua, J. M. Emulsion Polymerization: From Fundamental Mechanisms to Process Developments. J. Polym. Sci. Part Polym. Chem. 2004, 42 (5), 1025-1041.

(11) Asua, J. M. Challenges in Polymerization in Dispersed Media. In Polymer Reaction Engineering of Dispersed Systems: Volume II; Pauer, W., Ed.; Advances in Polymer Science; Springer International Publishing: Cham, 2018; pp 1-22.

(12) Jönsson, J. B.; Müllner, M.; Piculell, L.; Karlsson, O. J. Emulsion Condensation Polymerization in Dispersed Aqueous Media. Interfacial Reactions and Nanoparticle Formation. Macromolecules 2013, 46 (22), 9104-9113.

(13) Sousa, A. F.; Silvestre, A. J. D.; Gandini, A.; Neto, C. P. Synthesis of Aliphatic Suberinlike Polyesters by Ecofriendly Catalytic Systems. High Perform. Polym. 2012, 24 (1), 4-8.

(14) Baile, M.; Chou, Y. J.; Saam, J. C. Direct Polyesterifcation in Aqueous Emulsion. Polym. Bull. 1990, 23 (3), 251-257.

(15) Saam, J. C.; Huebner, D. J. Condensation Polymerization of Oligomeric Polydimethylsiloxanols in Aqueous Emulsion. J. Polym. Sci. Polym. Chem. Ed. 1982, 20 (12), 3351-3368.

(16) Takasu, A.; Takemoto, A.; Hirabayashi, T. Polycondensation of Dicarboxylic Acids and Diols in Water Catalyzed by Surfactant-Combined Catalysts and Successive Chain Extension. Biomacromolecules 2006, 7 (1), 6-9.

(17) Odian, G. Step Polymerization. In Principles of Polymerization; John Wiley \& Sons, Ltd, 2004; pp 39-197. https://doi.org/10.1002/047147875X.ch2.

(18) Billiet, L.; Fournier, D.; Du Prez, F. Step-Growth Polymerization and 'Click' Chemistry: The Oldest Polymers Rejuvenated. Polymer 2009, 50 (16), 3877-3886.

(19) Schork, F. J.; Luo, Y.; Smulders, W.; Russum, J. P.; Butté, A.; Fontenot, K. Miniemulsion Polymerization. In Polymer Particles; Springer, 2005; pp 129-255.

(20) Landfester, K.; Tiarks, F.; Hentze, H. P.; Antonietti, M. Polyaddition in Miniemulsions: A New Route to Polymer Dispersions. Macromol. Chem. Phys. 2000, 201 (1), 1-5. 
(21) Barrere, M.; Landfester, K. High Molecular Weight Polyurethane and Polymer Hybrid Particles in Aqueous Miniemulsion. Macromolecules 2003, 36 (14), 5119-5125.

(22) Barrère, M.; Landfester, K. Polyester Synthesis in Aqueous Miniemulsion. Polymer 2003, 44 (10), 2833-2841.

(23) Landfester, K.; Pawelzik, U.; Antonietti, M. Polydimethylsiloxane Latexes and Copolymers by Polymerization and Polyaddition in Miniemulsion. Polymer 2005, 46 (23), 9892-9898.

(24) Jasinski, F.; Rannee, A.; Schweitzer, J.; Fischer, D.; Lobry, E.; Croutxe-Barghorn, C.; Schmutz, M.; Le Nouen, D.; Criqui, A.; Chemtob, A. Thiol-Ene Linear Step-Growth Photopolymerization in Miniemulsion: Fast Rates, Redox-Responsive Particles, and Semicrystalline Films. Macromolecules 2016, 49 (4), 1143-1153.

(25) Jasinski, F.; Lobry, E.; Tarablsi, B.; Chemtob, A.; Croutxe-Barghorn, C.; Le Nouen, D.; Criqui, A. Light-Mediated Thiol-Ene Polymerization in Miniemulsion: A Fast Route to Semicrystalline Polysulfide Nanoparticles. Acs Macro Lett 2014, 3 (9), 958-962.

(26) Paulis, M.; Asua, J. M. Knowledge-Based Production of Waterborne Hybrid Polymer Materials. Macromol. React. Eng. 2016, 10 (1), 8-21.

(27) Asua, J. M. Miniemulsion Polymerization. Prog. Polym. Sci. 2002, 27 (7), 1283-1346..

(28) Asua, J. M. Challenges for Industrialization of Miniemulsion Polymerization. Prog. Polym. Sci. 2014, 39 (10), 1797-1826.

(29) Zhou, X.; Li, Y.; Fang, C.; Li, S.; Cheng, Y.; Lei, W.; Meng, X. Recent Advances in Synthesis of Waterborne Polyurethane and Their Application in Water-Based Ink: A Review. J. Mater. Sci. Technol. 2015, 31 (7), 708-722.

(30) Choi, K. Y.; McAuley, K. B. Step-Growth Polymerization. In Polymer Reaction Engineering; John Wiley \& Sons, Ltd, 2008; pp 273-314. https://doi.org/10.1002/9780470692134.ch7.

(31) Durham, O. Z.; Chapman, D. V.; Krishnan, S.; Shipp, D. A. Radical Mediated Thiol-Ene Emulsion Polymerizations. Macromolecules 2017, 50 (3), 775-783.

(32) Hoyle, C. E.; Bowman, C. N. Thiol-Ene Click Chemistry. Angew. Chem.-Int. Ed. 2010, 49 (9), 1540-1573. https://doi.org/10.1002/anie.200903924.

(33) Griesbaum, K. Problems and Possibilities of the Free-Radical Addition of Thiols to Unsaturated Compounds. Angew. Chem. Int. Ed. Engl. 1970, 9 (4), 273-287.

(34) Wang, S.; Daniels, E. S.; Sudol, E. D.; Klein, A.; El-Aasser, M. S. Isothermal Emulsion Polymerization of N-Butyl Methacrylate with KPS and Redox Initiators: Kinetic Study at Different Surfactant/Initiator Concentrations and Reaction Temperature. J. Appl. Polym. Sci. 2016, 133 (7), 1-8.

(35) Chern, C. S.; Lin, C. H. Using a Water-Insoluble Dye to Probe the Particle Nucleation Loci in Styrene Emulsion Polymerization. Polymer 1999, 40 (1), 139-147.

(36) Chern, C.; Lin, C. Particle Nucleation Loci in Emulsion Polymerization of Methyl Methacrylate. Polymer 2000, 41, 4473-4481.

(37) Nagarajan, R.; Chaiko, M. A.; Ruckenstein, E. Locus of Solubilization of Benzene in Surfactant Micelles. J. Phys. Chem. 1984, 88 (13), 2916-2922.

(38) Hanus, L. H.; Ploehn, H. J. Conversion of Intensity-Averaged Photon Correlation Spectroscopy Measurements to Number-Averaged Particle Size Distributions. 1. Theoretical Development. Langmuir 1999, 15 (9), 3091-3100. 
(39) Esfandiari, P.; Ligon, S. C.; Lagref, J. J.; Frantz, R.; Cherkaoui, Z.; Liska, R. Efficient Stabilization of Thiol-Ene Formulations in Radical Photopolymerization. J. Polym. Sci. Part Polym. Chem. 2013, 51 (20), 4261-4266.

(40) Belbakra, Z.; Cherkaoui, Z. M.; Allonas, X. Photocurable Polythiol Based (Meth) Acrylate Resins Stabilization: New Powerful Stabilizers and Stabilization Systems. Polym. Degrad. Stab. 2014, 110, 298-307.

(41) Edler, M.; Mostegel, F. H.; Roth, M.; Oesterreicher, A.; Kappaun, S.; Griesser, T. Enhancing the Stability of UV-Curable Thiol/Vinyl Carbonate Resins. J. Appl. Polym. Sci. 2017, 134 (24).

(42) Mengual, O.; Meunier, G.; Cayré, I.; Puech, K.; Snabre, P. TURBISCAN MA 2000: Multiple Light Scattering Measurement for Concentrated Emulsion and Suspension Instability Analysis. Talanta 1999, 50 (2), 445-456.

(43) Renz, P.; Kokkinopoulou, M.; Landfester, K.; Lieberwirth, I. Imaging of Polymeric Nanoparticles: Hard Challenge for Soft Objects. Macromol. Chem. Phys. 2016, 217 (17), 1879-1885.

(44) Elizalde, O.; Leal, G. P.; Leiza, J. R. Particle Size Distribution Measurements of PolymericDispersions: A Comparative Study. Part. Part. Syst. Charact. 2000, 17 (5-6), 236-243.

(45) Piradashvili, K.; Alexandrino, E. M.; Wurm, F. R.; Landfester, K. Reactions and Polymerizations at the Liquid-Liquid Interface. Chem. Rev. 2016, 116 (4), 2141-2169.

(46) Song, Y.; Fan, J.-B.; Wang, S. Recent Progress in Interfacial Polymerization. Mater. Chem. Front. 2017, 1 (6), 1028-1040.

(47) Nagarjuna, R.; Saifullah, M. S. M.; Ganesan, R. Oxygen Insensitive Thiol-Ene Photo-Click Chemistry for Direct Imprint Lithography of Oxides. RSC Adv. 2018, 8 (21), 1140311411.

(48) Ai, Z.; Deng, R.; Zhou, Q.; Liao, S.; Zhang, H. High Solid Content Latex: Preparation Methods and Application. Adv. Colloid Interface Sci. 2010, 159 (1), 45-59. 